\title{
Niveles de 25 hidroxivitamina $D$ y su correlación clínica con diferentes variables metabólicas y cardiovasculares en una población de mujeres posmenopáusicas
}

\author{
Levels of 25-hydroxyvitamin D and their \\ clinical correlation with several metabolic and \\ cardiovascular variables in a population of \\ postmenopausal women
}

José Fernando Molina, Javier Molina, Jorge Andrés Escobar, Juan Felipe Betancur, Andrea Giraldo • Medellín

\section{Resumen}

Introducción: la osteoporosis es la enfermedad ósea metabólica más común. Entre sus causas secundarias se encuentra la deficiencia de vitamina $\mathrm{D}(\mathrm{VD})$, la cual predispone además a fracturas por fragilidad e incrementa el riesgo de caídas. También confiere un riesgo incrementado de desarrollar enfermedad cardiovascular, diabetes mellitus tipo 1 y 2.

Objetivo: el objetivo principal del estudio fue determinar los niveles de vitamina D en la población y correlacionarlos con diferentes variables clínicas, de laboratorio y densitométricas.

Métodos: se realizó un estudio descriptivo de corte transversal, de una cohorte de pacientes donde se analizaron datos secundarios de mujeres posmenopáusicas colombianas con diagnóstico de osteoporosis y osteopenia $(\mathrm{N}=205)$. Se analizaron 46 variables donde se calcularon estadísticos descriptivos y regresiones lineales múltiples para determinar correlaciones.

Resultados: la prevalencia de niveles insuficientes de vitamina $\mathrm{D}$ fue $55.1 \%,(\mathrm{n}=113)$, deficientes $16.6 \%(n=34)$, y adecuados sólo $28.29 \%(n=58)$. Al comparar los pacientes con niveles deficientes e insuficientes, se encontró que los pacientes con niveles de vitamina $\mathrm{D}$ deficientes fue un factor de riesgo para la presencia de fracturas vertebrales, RR de 1.02 (IC: 0.96 a 1.06) y para la hipertensión arterial RR de 1.47 (IC: 1.36 a 1.58).

Conclusión: dos terceras partes de nuestra población de pacientes tienen niveles inadecuados de vitamina D, y se encontró correlación con fracturas vertebrales e hipertensión arterial (Acta Med Colomb 2011; 36: 18-23).

Palabras clave: osteoporosis, vitamina D, hipertensión, diabetes mellitus 2, densidad mineral ósea.

\footnotetext{
Abstract

Introduction: osteoporosis is the most common metabolic bone disease. Vitamin D deficiency is an important cause of secondary osteopenia and osteoporosis. It predisposes to fragility fractures and increases the risk of falling, while augmenting the risk of developing cardiovascular disease and diabetes mellitus type 1 and 2 .

Objective: the objective of this study was to determine the levels of vitamin D in our population study and to correlate them with bone density, vertebral fractures, and other cardiovascular and laboratory variables.

Methods: we conducted a cross-sectional study of a cohort $(n=205)$ of postmenopausal Colombian women diagnosed with osteoporosis and osteopenia. We analyzed 46 variables. Descriptive statistics were used, and multiple linear regressions were analyzed in order to determine correlations.

Results: it was found that the prevalence of insufficient levels of vitamin D was $55.1 \%(n=113)$, deficient levels $16.6 \%(n=34)$, and adequate levels in only $28.29 \%(n=58)$ of patients. Comparing poor
}

Trabajo original, realizado en el Centro Integral de Reumatología. REUMALAB (Medellín).

Presentación al XXI Congreso Colombiano de Medicina Interna 2010.

Dr. José Fernando Molina Restrepo: Internista, Reumatólogo, Director Científico; Dr. Javier Molina López: Internista, Reumatólogo, Director General; Dr. Jorge Andrés Escobar Marulanda: Jefe de Investigación; Dr. Juan Felipe Betancur Pulgarín: Médico Unidad de Investigación; Andrea Giraldo Serna: Gerente de Sistemas de Información en Salud, Coordinadora Investigación. Centro Integral de Reumatología. REUMALAB (Medellín).

Patrocinio financiero: NO.

Correspondencia: Dr. José Fernando Molina Restrepo, Carrera 48 No. $19^{\mathrm{a}}-40$ Consultorio 901. Edificio Torre Médica Ciudad del Rio

PBX: 4447901 Ext. 116 Fax: 4445901

E-mail: jfmolina@une.net.co jfmolina@reumalab.com

Recibido: 24/I/2011 Aceptado: 04/II/2011 
and inadequate levels, we found that deficient levels of vitamin D are a risk factor for vertebral fractures, with an RR of 1.02 (IC: 0,96 a 1.06) and for high blood pressure. with an RR of 1.47 (IC: 1.36 a 1.58).

Conclusions: our study shows that inadequate levels of vitamin D are common in our population. This is associated with low bone mass, vertebral fractures, and hypertension. Further studies are needed in our country to confirm our findings (Acta Med Colomb 2011; 36: 18-23).

Keywords: osteoporosis, vitamin D, hypertension, diabetes mellitus 2, bone mineral density.

\section{Introducción}

En Colombia la prevalencia promedio de osteoporosis es del $57 \%$ en la población mayor de 40 años, similar a la de otras poblaciones de Latinoamérica. Dentro de las múltiples causas secundarias de osteoporosis, se encuentra la deficiencia de vitamina $D$, ya que ésta interviene en el metabolismo óseo y puede contribuir a la patogénesis de la baja masa ósea y predisponer a fracturas por fragilidad (1-3).

Se ha encontrado de manera global que el $64 \%$ de las mujeres posmenopáusicas tienen hipovitaminosis D. Latinoamérica es la zona del mundo con menor deficiencia de VD (2, 4-6).

En Colombia se han realizado muy pocos estudios sobre prevalencia de niveles de vitamina $\mathrm{D}$ en mujeres posmenopáusicas y su relación con osteoporosis $(7,8)$.

Estudios clínicos recientes han demostrado que hay un incremento en el riesgo de desarrollar diabetes mellitus tipo 1 , diabetes mellitus tipo 2 y enfermedad cardiovascular en personas con niveles inadecuados de vitamina $\mathrm{D}$; que además tienen otros factores de riesgo asociados como hipertensión y obesidad $(7,8)$. Sin embargo, faltan estudios clínicos bien diseñados que permitan dilucidar de una manera más exacta esta causalidad, pues aún no se entienden del todo los mecanismos moleculares de esta asociación.

No hay consenso absoluto sobre el rango normal de los niveles séricos de vitamina $\mathrm{D}$, y en la actualidad existen diferentes rangos que difieren en los valores definidos, ya sea por consenso de expertos o de diferentes asociaciones mundiales (10-13).

La determinación actual de los niveles considerados como normales son basados en estudios donde se correlacionan la absorción intestinal de calcio y los niveles de PTH con VD (relación inversa). Los niveles de PTH alcanzan el nadir con una concentración de VD entre 30-40 ng/mL. Por lo tanto definiremos los niveles de 25 OHVD según el consenso de la mayoría de los expertos y según la respuesta fisiológica así:

- Adecuado o normal: > a $30 \mathrm{ng} / \mathrm{mL}$, este nivel se considerará como la meta por alcanzar en caso de inicio de suplencia.

- Deficiencia: niveles de $250 \mathrm{OHD}<=$ a $20 \mathrm{ng} / \mathrm{mL}$.

- Insuficiencia: niveles entre 20.1-29 ng/mL.

- Intoxicación: $>150 \mathrm{ng} / \mathrm{mL}$ que es asociado a los siguientes hallazgos de laboratorio: hipercalcemia, hipercalciuria e hiperfosfatemia (10-12, 14-16).

\section{Material y métodos}

El objetivo principal fue determinar los niveles de vitamina $\mathrm{D}$ en un grupo de pacientes posmenopáusicas colombia- nas con diagnóstico clínico y densitométrico de: baja masa osea u osteoporosis y correlacionar los valores de $\mathrm{T}$ score de columna, cuello femoral y la densidad mineral ósea, con los niveles de vitamina $\mathrm{D}$, entre otras variables de laboratorio.

Además correlacionar estos valores de vitamina $\mathrm{D}$, con variables cardiovasculares.

Para dar cumplimiento a estos objetivos se realizó un estudio descriptivo de corte transversal, de una cohorte de pacientes que participaron en un ensayo clínico doble ciego aleatorizado, la población de estudio fue de 298 donde se tomó una muestra de 205 pacientes, se utilizó un alfa de $(\alpha)$ 0.04 con un $\mathrm{Z}$ de 2,053 , un nivel de confianza del $96 \%$ para el cálculo del tamaño de muestra. La muestra se escogió mediante un muestreo aleatorio simple. En el ensayo clínico se incluyeron pacientes que cumplían con los siguientes criterios de inclusión:

Paciente sexo femenino, entre 60 y 85 años de edad; con una expectativa de vida mayor de cinco años (según criterio médico); último periodo menstrual mínimo dos años antes de ingresar al estudio; consentimiento informado diligenciado y firmado por el paciente, dos testigos y el investigador; tener dos vértebras evaluables por DXA y rayos $\mathrm{X}$ de columna lateral al momento del screening. T score menor o igual a $-1.0 \mathrm{al}$ momento del screening.

\section{Criterios de exclusión}

Paciente con desorden metabólico diferente a osteoporosis o baja densidad mineral ósea (hipertiroidismo, osteodistrofia renal, u osteomalacia).

Se tomaron 46 variables que habían sido recolectadas en un estudio clínico (datos secundarios), en donde se tienen variables demográficas y clínicas. Con estas variables se calcularon estadísticos descriptivos de tendencia central y de dispersión, además se realizó una regresión lineal múltiple para determinar la relación entre los valores de T score de columna, cuello femoral, y densidad mineral ósea, con el resto de las variables.

Así mismo, se establecieron medidas de asociación entre variables cualitativas (riesgo relativo).

Se planteó un modelo de regresión lineal en el cual se determinaron las variables independientes que mejor predicen el resultado de la variable dependiente, para poder observar la correlación existente entre éstas (método enter). El análisis estadístico se realizó bajo el paquete SPSS Versión 15.

\section{Resultados}

La Tabla 1 muestra las características más importantes de las pacientes que participaron en el estudio. 
Tabla 1. Características del total de pacientes, pacientes con niveles de vitamina $D$ adecuados y con niveles de vitamina $D$ inadecuados.

\begin{tabular}{|c|c|c|c|}
\hline & $\begin{array}{l}\text { Total grupo } \\
\quad(n=205)\end{array}$ & $\begin{array}{l}\text { Niveles de vitamina D adecuados } \\
\qquad(\mathrm{n}=58)\end{array}$ & $\begin{array}{l}\text { Niveles de vitamina } D \text { inadecuados } \\
\qquad(n=147)\end{array}$ \\
\hline Edad (años) & $66.6 \pm 4.8$ & $67.3 \pm 5.2$ & $66.3 \pm 4.7$ \\
\hline Peso $(\mathrm{kg})$ & $58.9 \pm 9.1$ & $58.1 \pm 8.3$ & $59.3 \pm 9.4$ \\
\hline Talla (m) & $1.5 \pm 0.057$ & $1.5 \pm 0.05$ & $1.5 \pm 0.06$ \\
\hline Circunferencia abdominal $(\mathrm{cm})$ & $90.8 \pm 12.5$ & $89.7 \pm 11.4$ & $91.2 \pm 12.7$ \\
\hline Índice de masa corporal $\left(\mathrm{kg} / \mathrm{m}^{2}\right)$ & $26.1 \pm 3.7$ & $25.7 \pm 2.7$ & $26.7 \pm 3.9$ \\
\hline T score columna & $-2.8 \pm 0.99$ & $-2.8 \pm 1.2$ & $-2.7 \pm 0.9$ \\
\hline T score fémur & $-2.2 \pm 0.72$ & $-2.2 \pm 0.62$ & $-2.1 \pm 0.8$ \\
\hline Densidad mineral ósea $\left(\mathrm{g} / \mathrm{cm}^{2}\right)$ & $0.7 \pm 0.11$ & $0.73 \pm 0.12$ & $0.8 \pm 0.1$ \\
\hline Valor de vitamina $\mathrm{D}(\mathrm{ng} / \mathrm{mL})$ & $26.7 \pm 6.89$ & $35.4 \pm 4.5$ & $23.3 \pm 3.9$ \\
\hline Valor de creatinina (umol/L) & $73.1 \pm 12.7$ & $74.2 \pm 15.5$ & $72.7 \pm 11.5$ \\
\hline Valor de calcio $(\mathrm{mmol} / \mathrm{L})$ & $2.5 \pm 0.09$ & $2.6 \pm 0.08$ & $2.5 \pm 0.09$ \\
\hline Valor de fósforo $(\mathrm{mmol} / \mathrm{L})$ & $1.3 \pm 0.14 \pm$ & $1.3 \pm 0.14$ & $1.3 \pm 0.1$ \\
\hline
\end{tabular}

El valor medio de los niveles de vitamina $\mathrm{D}$ encontrado para las 205 pacientes fue de $26.7 \mathrm{ng} / \mathrm{mL}$, por su parte para las pacientes con niveles inadecuados el valor medio fue de $23.9 \mathrm{ng} / \mathrm{mL}$. La prevalencia de niveles inadecuados es de $71.71 \%(\mathrm{n}=147)$ incluyendo valores insuficientes $55.1 \%$ $(\mathrm{n}=113)$, y deficientes de vitamina D 16.6\% $(\mathrm{n}=34)$.

De los 205 pacientes, un $40 \%(\mathrm{n}=82)$ presentaba hipertensión (HTA), un 8\% ( $n=17)$ diabetes, un $32 \%(n=65)$ fumaba y el $3 \%(n=7)$ consumía alcohol. Para el grupo de pacientes con niveles de vitamina $\mathrm{D}$ adecuados, un $34 \%$ $(n=20)$ de las pacientes eran hipertensas, mientras que las pacientes con niveles inadecuados, un $42 \%(n=62)$ presentan esta patología. Ciento treinta pacientes presentaron fracturas vertebrales con un total de 269 fracturas, donde 252 tenían un grado leve, 15 fracturas con grado moderado y sólo dos fracturas tenían un grado severo. Para las pacientes con niveles de vitamina $\mathrm{D}$ insuficientes se encontró que ocho pacientes tenían fracturas moderadas y 133 fracturas leves.
Por estos datos podemos inferir que factores como el tabaquismo y el consumo de alcohol podrían intervenir en los niveles de vitamina $\mathrm{D}$.

En pacientes con comorbilidades como diabetes mellitus 2 , hipertensión arterial y fracturas vertebrales, se encuentra una mayor frecuencia de niveles inadecuados de vitamina D (Tabla 3).

Al comparar los pacientes con niveles deficientes e insuficientes se encontró que los niveles de vitamina $\mathrm{D}$ deficientes son un factor de riesgo para la presencia de fracturas vertebrales, con un RR de 1.02 (IC: 0.96 a 1.06 ) y para la hipertensión arterial, con un RR de 1.47 (IC: 1.36 a 1.58). El riesgo de presentar fracturas cuando se tiene niveles inadecuados de vitamina $D$ es dos veces más en comparación con pacientes con niveles de vitamina $\mathrm{D}$ adecuados, por su parte el riesgo de presentar hipertensión es tres veces más en pacientes con niveles inadecuados que en los pacientes con niveles adecuados de vitamina D.

Tabla 2. Distribución de frecuencia del los pacientes HTA, diabetes, paciente que consumen alcohol, tabaco, presencia de fracturas vertebrales y grado de las fracturas vertebrales.

\begin{tabular}{|c|c|c|c|c|c|}
\hline & Todos & Adecuados & Inadecuados & Deficientes & Insuficientes \\
\hline HTA & $82(40 \%)$ & $20(34 \%)$ & $62(42 \%)$ & $19(13 \%)$ & $43(29 \%)$ \\
\hline Diabéticos & $17(8 \%)$ & $4(7 \%)$ & $13(9 \%)$ & $6(4 \%)$ & $7(5 \%)$ \\
\hline Fuman & $65(32 \%)$ & $16(28 \%)$ & $49(33 \%)$ & $13(9 \%)$ & $36(24 \%)$ \\
\hline Alcohol & $7(3 \%)$ & $0(0 \%)$ & $7(4 \%)$ & $1(1 \%)$ & $6(4 \%)$ \\
\hline Pacientes con fractura vertebral & $130(63 \%)$ & $40(20 \%)$ & $90(44 \%)$ & $21(23 \%)$ & $69(77 \%)$ \\
\hline Total pacientes & 205 & 58 & 147 & 34 & 113 \\
\hline
\end{tabular}

Tabla 3. Distribución de frecuencia del número de fracturas vertebrales y grado de la fractura, según los niveles de vitamina D.

\begin{tabular}{|l|c|c|c|c|}
\hline & Todos & Adecuados & Inadecuados & Deficientes \\
\hline No. Frac. Vert. Pres. & 269 & 81 & 188 & 47 \\
No. Frac. leves & 252 & 74 & 178 & 141 \\
No. Frac. moderadas & 15 & 5 & 10 & 0 \\
No. Frac. severas & 2 & 2 & 0 \\
\hline
\end{tabular}


Al realizar la regresión lineal múltiple tomando como variable dependiente el valor de $\mathrm{T}$ score de columna, en pacientes con niveles de vitamina $\mathrm{D}$ deficientes, el modelo nos sugiere que los cambios presentados en este valor se explican en un $95.2 \%$, por las variables independientes (***). Esto quiere decir que por cada cambio en el valor del T score de columna, un $95 \%$ se debe a las variables independiente, el modelo es estadísticamente significativo al obtener un valor de $P$ inferior a 0.05 , además en los coeficientes de regresión parcial ninguna de las variables independientes contiene el cero en sus intervalos de confianza (Tabla 4).

Cuando se toma como variable dependiente el valor de $\mathrm{T}$ score de cuello femoral, para las pacientes con niveles de vitamina $\mathrm{D}$ deficientes, el modelo nos sugiere que los cambios presentados en el valor del $\mathrm{T}$ score de cuello femoral se explican en un $45.1 \%$, por la variables independientes (*****). El modelo obtuvo un valor de P de 0.02 (Tabla 5). Cuando la variable dependiente es la densidad mineral ósea, para las pacientes con niveles de vitamina D inadecuados, el modelo nos sugiere que los cambios presentados en el valor de la densidad mineral ósea se explican en un
$79.5 \%$, por las variables independientes $(* * * *)$, valor de p 0.00 (Tabla 6).

Los resultados del modelo de regresión lineal múltiple demuestran que entre las variables independientes que predicen la variable dependiente (La DMO, el T score de columna y fémur) se encuentran los niveles inadecuados de vitamina D.

\section{Discusión}

En Colombia se han realizado pocos estudios sobre la prevalencia de niveles de vitamina $\mathrm{D}$ en mujeres posmenopáusicas y su relación con osteoporosis. Los que se han hecho poseen muestras muy pequeñas (7) o miden metabolitos que se consideran inadecuados para valorar los niveles de vitamina $\mathrm{D}(8)$; según datos de estos estudios en nuestra población los niveles promedio de vitamina D son de $32.68 \mathrm{ng} / \mathrm{mL}$, y sólo sus niveles se encuentran $33 \%$ disminuidos entre las mujeres osteopénicas respecto a las mujeres con DMO normal (7). Contrario a lo observado en éstos, el presente estudio indica que los niveles inadecuados de vitamina D tienen una alta prevalencia $(71.71 \%$ inclu-

Tabla 4. Análisis de regresión lineal múltiple de las variables que afectan el valor de T score de columna en mujeres posmenopáusicas.

\begin{tabular}{|c|c|c|c|c|c|c|}
\hline \multicolumn{2}{|c|}{ T score de columna } & Todos* & Adecuados* & Inadecuado** & Insuficientes $* *$ & Deficientes ${ }^{* * *}$ \\
\hline \multicolumn{2}{|l|}{$\mathrm{R} 2$} & 0.811 & 0.797 & 0.838 & 0.82 & 0.96 \\
\hline \multicolumn{2}{|c|}{ R 2 corregido } & 80.7 & 78.9 & 83.2 & 81.2 & 95.2 \\
\hline \multicolumn{2}{|l|}{ SIG } & 0.00 & 0.00 & 0.00 & 0.00 & 0.00 \\
\hline \multicolumn{7}{|c|}{$\begin{array}{l}\text { * Variables independientes: el valor de la creatinina en el examen de laboratorio de la primera visita, el valor de } \mathrm{T} \text { score de cuello femoral, el valor de la vitamina } \mathrm{D} \text { en la primera } \\
\text { visita dividido por } 2.5 \mathrm{y} \text { el valor de la densidad mineral ósea. }\end{array}$} \\
\hline \multicolumn{7}{|c|}{$\begin{array}{l}\text { ** Variables independientes: el valor de la vitamina } \mathrm{D} \text { en la primera visita dividido por } 2.5 \text {, el valor de la circunferencia abdominal, La edad del paciente al momento de ingresar } \\
\text { al estudio, el valor del T score de cuello femoral y el valor de la densidad mineral ósea. }\end{array}$} \\
\hline \multicolumn{7}{|c|}{$\begin{array}{l}\text { ** Variables independientes: es el valor del calcio en el examen de laboratorio de la primera visita, es el valor de la vitamina D en la primera visita dividido por } 2.5 \text {, circunferencia } \\
\text { abdominal, densidad mineral ósea., Es el valor de la creatinina en el examen de laboratorio de la primera visita. }\end{array}$} \\
\hline
\end{tabular}

Tabla 5. Análisis de regresión lineal múltiple de las variables que afectan el valor de T score de fémur en mujeres posmenopáusicas.

\begin{tabular}{|c|c|c|c|c|c|c|}
\hline \multicolumn{2}{|c|}{$T$ score de fémur } & Todos* & Adecuados** & Inadecuado**** & Insuficientes $* * * *$ & Deficientes $* * * * *$ \\
\hline \multicolumn{2}{|l|}{$\mathrm{R} 2$} & 0.319 & 0.373 & 0.341 & 0.378 & 0.571 \\
\hline \multicolumn{2}{|c|}{ R 2 corregido } & 29.1 & 28.3 & 31.7 & 34.8 & 45.1 \\
\hline \multicolumn{2}{|l|}{ SIG } & 0.00 & 0.01 & 0.00 & 0.00 & 0.00 \\
\hline \multicolumn{7}{|c|}{$\begin{array}{l}\text { * Variables independientes: el valor de la creatinina en el examen de laboratorio de la primera visita, el valor de la vitamina } \mathrm{D} \text { en la primera visita dividido por } 2.5 \text {, el valor de la } \\
\text { circunferencia abdominal, la edad del paciente al momento de ingresar al estudio, la talla del paciente al momento de ingresar al estudio, el valor de T score de columna, el valor } \\
\text { del índice de masa corporal y el peso de la paciente al momento de ingresar al estudio. }\end{array}$} \\
\hline$* *$ & \multicolumn{6}{|c|}{$\begin{array}{l}\text { Variables independientes: el valor del fósforo en el examen de laboratorio de la primera visita, el valor de la circunferencia abdominal, el valor de la vitamina D en la primera visita } \\
\text { dividido por } 2.5 \text {, el valor de la creatinina en el examen de laboratorio de la primera visita, la edad del paciente al momento de ingresar al estudio, el valor de } \mathrm{T} \text { score de columna, } \\
\text { el valor del índice de masa corporal. }\end{array}$} \\
\hline$* * *$ & \multicolumn{6}{|c|}{$\begin{array}{l}\text { Variables independientes: el valor de la vitamina } \mathrm{D} \text { en la primera visita dividido por } 2.5 \text {, el valor de la circunferencia abdominal, la talla del paciente al momento de ingresar al } \\
\text { estudio, el valor de T score de columna, el peso de la paciente al momento de ingresar al estudio }\end{array}$} \\
\hline$* * * *$ & \multicolumn{6}{|c|}{$\begin{array}{l}\text { Variables independientes: el valor de la vitamina } \mathrm{D} \text { en la primera visita dividido por } 2.5 \text {, el valor de la circunferencia abdominal, la edad del paciente al momento de ingresar al } \\
\text { estudio, el valor de T score de cuello femoral, el valor de la densidad mineral ósea. }\end{array}$} \\
\hline$* * * * *$ & \multicolumn{6}{|c|}{$\begin{array}{l}\text { Variables independientes: es el valor del fósforo en el examen de laboratorio de la primera visita, es el valor de la creatinina en el examen de laboratorio de la primera visita, es la } \\
\text { edad del paciente al momento de ingresar al estudio, es el peso de la paciente al momento de ingresar la estudio, es el valor de la vitamina } D \text { en la primera visita dividido por } 2.5 \text {, } \\
\text { T score de columna, densidad mineral ósea. }\end{array}$} \\
\hline
\end{tabular}


Tabla 6. Análisis de regresión lineal múltiple de las variables que afectan el valor de la densidad mineral ósea en mujeres posmenopáusicas.

\begin{tabular}{|c|c|c|c|c|c|c|}
\hline \multicolumn{2}{|l|}{ DMO } & Todos* & Adecuados** & Inadecuado*** & Insuficientes $* * * *$ & Deficientes $* * * * *$ \\
\hline \multicolumn{2}{|l|}{ R 2} & 0.802 & 0.782 & 0.341 & 0.805 & 0.967 \\
\hline \multicolumn{2}{|c|}{ R 2 corregido } & 79.6 & 76.6 & 31.7 & 79.5 & 95.7 \\
\hline \multicolumn{2}{|l|}{ SIG } & 0.00 & 0.00 & 0.00 & 0.00 & 0.00 \\
\hline \multicolumn{7}{|c|}{$\begin{array}{l}\text { * Variables independientes: el valor del fósforo en el examen de laboratorio de la primera visita, el valor de la vitamina } \mathrm{D} \text { en la primera visita dividido por } 2.5 \text {, el valor de } \mathrm{T} \text { score } \\
\text { de columna, el valor del calcio en el examen de laboratorio de la primera visita, el valor de la creatinina en el examen de laboratorio de la primera visita, el peso de la paciente al } \\
\text { momento de ingresar al estudio. }\end{array}$} \\
\hline$* *$ & \multicolumn{6}{|c|}{$\begin{array}{l}\text { Variables independientes: el valor del calcio en el examen de laboratorio de la primera visita, la edad del paciente al momento de ingresar al estudio, el valor de T score de columna, } \\
\text { el valor de la vitamina D en la primera visita dividido por } 2.5\end{array}$} \\
\hline$* * *$ & \multicolumn{6}{|c|}{$\begin{array}{l}\text { Variables independientes: es el valor del fósforo en el examen de laboratorio de la primera visita, el valor de T score de columna, el valor de la creatinina en el examen de laboratorio } \\
\text { de la primera visita, la edad del paciente al momento de ingresar al estudio, el valor de la vitamina D en la primera visita dividido por } 2.5 \text {, el peso de la paciente al momento de } \\
\text { ingresar al estudio }\end{array}$} \\
\hline$* * * *$ & \multicolumn{6}{|c|}{$\begin{array}{l}\text { Variables independientes: el valor del fósforo en el examen de laboratorio de la primera visita, el peso de la paciente al momento de ingresar al estudio, el valor de la vitamina } \mathrm{D} \\
\text { en la primera visita dividido por } 2.5 \text {, es el valor de la creatinina en el examen de laboratorio de la primera visita, el valor de } \mathrm{T} \text { score de columna. }\end{array}$} \\
\hline$* * * * *$ & \multicolumn{6}{|c|}{$\begin{array}{l}\text { Variables independientes: es el valor del calcio en el examen de laboratorio de la primera visita, el valor de la vitamina } \mathrm{D} \text { en la primera visita dividido por } 2.5 \text {, el valor de la cir- } \\
\text { cunferencia abdominal, el valor de } \mathrm{T} \text { score de cuello femoral, el valor de la creatinina en el examen de laboratorio de la primera visita, el valor de } \mathrm{T} \text { score de columna, el peso de } \\
\text { la paciente al momento de ingresar al estudio. }\end{array}$} \\
\hline
\end{tabular}

yendo valores insuficientes $55.1 \%$, y deficientes de vitamina D $16.6 \%$ ) en una población de mujeres posmenopáusicas osteoporóticas y con baja masa ósea de una población de la ciudad de Medellín.

Así mismo el parámetro para medir los niveles de vitamina D es la 25OHVD, un metabolito fácil de medir, disponible en el país, que debe tenerse en cuenta en el estudio de la paciente con osteoporosis, en particular en sospecha de causa secundaria (24). Existen múltiples discrepancias actualmente entre expertos y entre asociaciones acerca de los niveles adecuados e inadecuados de vitamina $\mathrm{D}$, una aproximación lógica y plausible fisiológicamente es la que correlaciona inversamente éstos con el nivel de PTH $(<30$ $\mathrm{ng} / \mathrm{mL})$.

Con base en estos resultados, como en lo descrito en la literatura mundial, sugerimos que la concentración de vitamina $\mathrm{D}$ por debajo de los rangos normales favorece la presentación de osteoporosis, y se correlaciona con alteración de los hallazgos densitométricos.

Múltiples estudios observacionales han mostrado una relación entre bajos niveles de vitamina $\mathrm{D}$ e hipertensión arterial (HTA), y enfermedad cardiovascular (ECV) existente, hallazgos que correlacionan con lo observado en nuestra cohorte. Por otro lado, estudios de modelos animales y humanos sugieren que la vitamina D realiza un papel importante en la homeostasis del metabolismo de la glucosa y el desarrollo de DM1 y DM2. Así mismo se ha demostrado la presencia de receptores de vitamina $\mathrm{D}$ en el músculo liso vascular (11).

Medellín localizada en Colombia (América del Sur) situada en la latitud de norte $06^{\circ} 17^{\prime} 19,25^{\prime}$ " y en la longitud de oeste $075^{\circ} 33^{\prime} 44,71$ " (25) es una ciudad ubicada cerca del Ecuador, sin variación estacional, y por lo tanto con mayor incidencia de rayos UVB de longitud de onda apropiada para la producción epidérmica de vitamina D; a

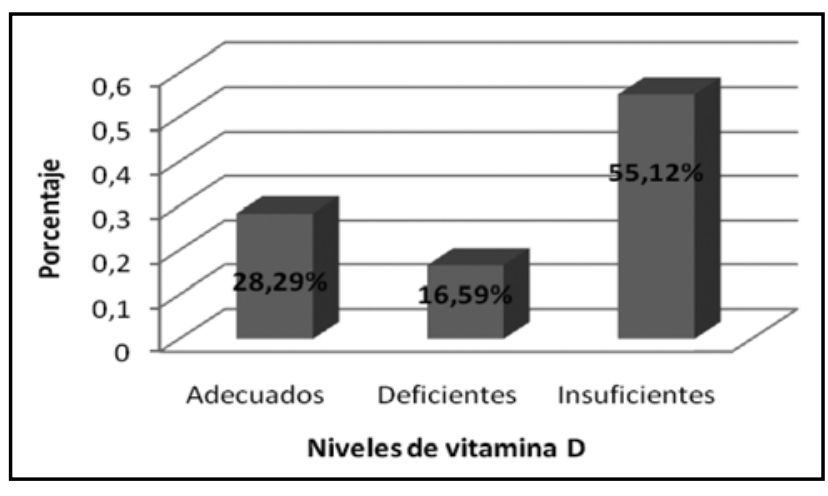

Figura 1. Prevalencia de los niveles de vitamina $D(n=205)$.

pesar de ésto, vemos que al parecer los niveles de vitamina D pueden estar influenciados por características en estilo de vida y ambientales (marcadores surrogados predictores de niveles de vitamina D) $(4,26,27)$.

La suplementación con vitamina $\mathrm{D}$ en el manejo de la osteoporosis y prevención de riesgo cardiovascular tiene un gran potencial en las políticas de salud pública, por su bajo costo, excelente tolerabilidad y beneficios combinados musculoesqueléticos. La evidencia disponible, las opiniones de expertos y las recomendaciones de las principales asociaciones mundiales de osteoporosis sugieren que la dosis de vitamina $\mathrm{D}$ en el manejo de la osteoporosis y prevención de caídas y fracturas no debe ser menor de 700-800 UI (28-30). Dosis menores de 600 se consideran ineficaces e insuficientes y los niveles de 25 OHVD mayores de $30 \mathrm{ng} /$ mL no serán probablemente alcanzados (28-30).

Con respecto a la dosis de suplementacion en prevención de riesgo cardiovascular, se han planteado dosis entre 1000 y 2000 UI día, aunque faltan aún más estudios concluyentes, que nos permitan definir una dosis exacta. Por lo tanto se debe considerar la suplementación de la vitamina $\mathrm{D}$ en 
el manejo de la paciente posmenopáusica con osteopenia/ osteoporosis, además en las pacientes con algún riesgo cardiovascular, ya que ésta es una medida costo efectiva en el manejo de nuestras pacientes.

En conclusión, nuestro estudio demuestra que los niveles inadecuados de vitamina D son frecuentes en nuestra población de pacientes, lo cual se asocia a baja masa ósea, fracturas vertebrales e hipertensión arterial. Se requieren más estudios en nuestro país que confirmen nuestros hallazgos.

\section{Agradecimientos}

Se agradece al doctor Jorge Hernando Donado Gómez por la revisión y corrección del artículo.

\section{Referencias}

1. Franco CB, Paz G, Gomes PE, Nascimento VB, Kulak CAM, Boguszewski CL, et al. Chronic obstructive pulmonary disease is associated with osteoporosis and low levels of vitamin D. Osteoporos Int 2009;20 (11):1881-7.

2. Carmona F. Osteoporosis en Santa Fe de Bogotá. Instituto Nacional de Salud. 1999.

3. Isenor JE, Emson MH. Is There a Role for Therapeutic Drug Monitoring of Vitamin D Level as a Surrogate Marker for Fracture Risk? Pharmacotherapy 2010;30 (3):254-64.

4. Quintana M, Toro C, Iglesias A. Historia de la Vitamina D. Revista Colombiana de Osteologia y Metabolismo Mineral 2004;3(1):32-54.

5. Rizzoli R, Eisman JA, Norquist J, Ljunggren O, Krishnarajah G, Lim SK, et al. Risk factors for vitamin D inadequacy among women with osteoporosis: an international epidemiological study. Int J Clin Pract 2007;60(8):1013-9.

6. Mithal A, Wahl DA, Bonjour JP, Burckhardt P, Dawson-Hughes B, Eisman JA, et al. Global vitamin D status and determinantsof hypovitaminosis D. Osteoporos Int 2009;20:1807-20.

7. Chavez RY, Garzon N, Wittingham E, Estrada R, Onatra W, Posso H. Determinación de los niveles de vitamina D y su relación en la Densidad Mineral Ósea (DMO) en mujeres peri-menopáusicas. Revista de Menopausia 2002;8(2).

8. Guevara M, Mogollón L, Iglesias A, Yupanqui H, Bermúde A. Estimación de Vitamina D en mujeres con osteopenia y osteoporosis en Cundinamarca-Colombia, por medio de extracción en fase sólida, cromatografía líquida de alta resolución y análisis multivariado. Nova- Publicación cientifica 2003;1(1):1-116.

9. Zittemann A, Schleithoff SS, Koerfer R. Putting cardiovascular disease and vitamin D insufficiency into perpective. Br J Nutr 2005;94(4):483-92.

10. Holick M. Vitamin D status: measurement, interpretation and clinical application. Ann Epidemiol 2009;19(2):73-8.

11. Kulie T, Groff A, Redmer J, Hounshell J, Schrager S. Vitamin D: An EvidenceBased Review. J Am Board Fam Med 2009;22:698 -706.
12. Wolff A, Jones A, Hnasen K. Vitamin D and musculoskeletal health. Nat Clin Pract Rheumatol 2008;4(11):580-8.

13. Ginanjar E, Setiati S, Setiyohadi B, Sumariyono. Vitamin D and autoinmune diseases. Acta Med Indones 2007;39(3):133-41.

14. Holick M. Vitamin D deficiency. N Engl J Med 2007;357:266-81.

15. Heaney R. The vitamin D requirement in health and disease. J Steroid Biochem Mol biol 2005;97(1-2):13-9.

16. Hansen K. Revalence of hypovitaminosis d and relationship to fracture nature. Reviews Rheumatology 2009;5:417-8.

17. Bischoff-ferrari HA, Dietrich T, Orav EJ, Dawson-Hughes B. Positive association between 25 -hydroxy vitamin $\mathrm{D}$ levels and bone mineral density: a populationbased study of younger and older adult. The Am J Med 2004;116:634-9.

18. Dawson-Hughes B, Heaney RP, Holick MF, Lips P, Meunier PJ, Vieth R. Estimates of optimal vitamin d status. Osteoporos Int 2005;16(7):713-6.

19. Hoeck HC, Li B. Qvist P. Changes in 25-hydroxyvitamin D3 to oral treatment with vitamin $\mathrm{d} 3$ in postmenopausal females with osteoporosis. Osteoporos Int 2009;20:1329-35.

20. Bischoff-Ferrari HA, Giovannucci E, Willett WC, Dietrich T, Dawson-Hughes B, Angel L, et al. Estimation of optimal serum concentrations of 25-hydroxyvitamin D for multiple Health outcomes. Am J Clin Nutr 2006;84:18-28.

21. Dawson-Hughes B, Harris SS, Krall EA, Dallal G. Effect of calcium and vitamin D supplementation on bone density in men and women 65 years of age or older. $\mathrm{NEngl}$ J Med 1997;337:670-6.

22. Chapuy MC, Arlot ME, Duboeuf F, J Brun, B Crouzet, S Arnaud, et al. Vitamin D3 and calcium to prevent hip fractures in the elderly women. $N$ Engl $J$ Med 1992;327:1637-42.

23. Ooms ME, Roos JC, Bezemer PD, Van Der Vijgh WJ, Bouter IM, Lips P. Prevention of bone loss by vitamin D supplementation in elderly women: a randomized double-blind trial. J Clin Endocrinol Metab 1995;80:1052- 8.

24. Gonzalez LA, Vasquez GM, Molina JF. Epidemiología de la osteoporosis. Revista Colombiana de Reumatologia 2009;16(1):61-75.

25. Tecnologies E. Mapa satélite de Medellín - Colombia. Journal [serial on the Internet]. 2010 Date: Available from: http://www.spanishcourses.info/citiesMAP/ medellin-colombia-mapa-270-ES.htm.

26. Millen AE, Wactawski-Wende J, Pettinger M, Melamed ML, Tylavsky FA, Liu S, et al. Predictors of serum 25-hydroxyvitamin D concentrations among postmenopausal women: the women's health initiative calcium plus vitamin $\mathrm{d}$ clinical. Am J Clin Nutr 2010;91(5):1324-35.

27. Rockell JE, Skeaff CM, Williams SM, Green T. Association between quantitative measures of skin color and plasma 25-hydroxyvitamin D. Osteoporos Int 2008; 19:1639-42.

28. Bischoff H. How to select the doses of vitamin D in the management of osteoporosis. Osteoporos Int 2007;18:401-7.

29. National Osteoporosis Foundation. Clinicians Guide to Prevention and Treatment of Osteoporosis. Journal [serial on the Internet]. 2008 Date August 22 2009]: Available from: http://www.nof.org/professionals/NOFCliniciansGuide.pdf.

30. Brown JP, Josse RG; Scientific Advisory Council of the Osteoporosis Society of Canada. 2002 clinical practice guidelines for the diagnosis and management of osteoporosis in Canada. CMAJ 2002;167:S1-34. 